\title{
Optimal design of reinforced concrete structures taking into account the particular calculation for progressive destruction
}

\author{
Ashot Tampazyan $^{* 1}$ and Svetlana Zubareva ${ }^{* * 1}$ \\ ${ }^{1}$ Moscow state university of Civil Engineering, 129337, 26, Yaroslavskoe shosse, Moscow, Russian \\ Federation.
}

\begin{abstract}
The problems of optimization of reinforced concrete structures are considered taking into account their properties and features under fire impacts and dynamic loading.

Experimental data on the dynamic strength of concrete and reinforcement under fire impacts and their approximation in analytical form are presented. It is shown that the dynamic strength of materials at temperatures above $3500 \mathrm{C}$ leads to their sharp decrease, which adversely affects the resistance of buildings to progressive destruction.

To resist the progressive destruction of buildings and structures, they require a high cost of providing them, which in most cases is inefficient. The search method is proposed, which allows taking into account a set of constraints, and the objective function, including the risk of collapse, to find the optimal solution.

On the example of a reinforced concrete bezel plate of overlap, its optimum parameters are found.

Keywords. Optimization, objective function, constraint system, risk analysis, search method, progressive destruction, fire effects, dynamic impact, dynamic coefficient, beamless slab
\end{abstract}

\section{Introduction}

Nowadays, special attention is paid to the problem of ensuring the safety of buildings and structures from progressive and other types of destruction, drawing back the issues of their optimal design [1]

The traditional design method in these conditions does not allow to reveal significant reserves of saving materials and structures. Economic efficiency can be significantly increased by optimizing cross sections and reinforcing structural elements [2]. The economic approach to optimization problems is reduced to constructing the objective function of the cost of the construction and finding its minimum.

\section{Results and discussion}

When calculating for progressive destruction, the objective function F2 is found as the sum of the costs, taking into account its manufacture, operation, necessary to maintain a certain level of reliability throughout the service life, the volume of restoration work, the risk of failure, the risk of failure, and the cost of elimination of consequences of possible emergency situations.

\footnotetext{
*tamrazian@mail.ru

**zubareva494@mail.ru
} 


$$
F_{2}=k_{c} k_{z}\left[C_{1} V_{b}+C_{2} V_{b}^{2}+\sum_{r}\left(C_{3} V_{s}+C_{4} V_{s}^{2}\right)+\sum_{i} C_{a d}+\left(1-P_{t}\right) \cdot C_{s e r}+R(t)\right],
$$

where $k_{c}$ - coefficient of conversion;

$k_{z}$ - coefficient of winter appreciation;

$C_{l}$ - production cost of concrete for $1 \mathrm{~m}^{3}$;

$V_{b}$ - volume of concrete;

$C_{2}$ - production cost of reinforcing steel for $1 \mathrm{~m}^{3}$;

$V_{s}$ - volume of reinforcing steel of the given type;

$\sum \mathrm{C}_{\mathrm{ad}}$-additional costs, including the consumption of reinforcement on the framing of technological holes, overlapping and anchoring;

$\left(1-P_{t}\right) \cdot C_{s \mathrm{e} r}$ - operational costs in view of the probability of failure-free operation of the structure;

$R(t)$ - loss risk arising from a design failure.

$$
R(t)=C_{y} \cdot Q
$$

$Q$ - probability of design failure;

$C_{y}$ - potential damage in case of failure of structures.

Compared to the function

$$
F_{1}=k_{c} k_{z}\left[C_{1} V_{b}+C_{2} V_{b}^{2}+\sum_{r}\left(C_{3} V_{s}+C_{4} V_{s}^{2}\right)+\sum_{i} C_{a d}\right],
$$

taking into account only the initial constraints corresponding to the limiting states of CП 63.13330.2012 (limiting bending moments, forces, deflections, crack opening width, etc.), it is clear that a fundamentally new approach is required to solve it.

With optimal design, the system of constraints will complement the following positions:

- restrictions on the expenditure of resources that must be established, based on the level of funding, or by analogy with previously known solutions. This includes restrictions on the amount of concrete, reinforcement, labor, energy costs, etc. The restrictions of this group have the form

$$
V_{b} \leq\left[V_{b}\right], G_{s} \leq\left[G_{s}\right], T \leq[T], \ldots
$$

- $\quad$ Constructive, architectural, technological limitations that significantly affect both the appearance of the structure and the shape or size of its elements

$$
\varphi_{k} \leq[\varphi]_{k}, k=1,2, \ldots
$$

- $\quad$ Restriction on the coefficient of renovation during repair work:

$$
k_{n}=\frac{C_{s e r}}{F_{K}} \leq\left[k_{n}\right]
$$

$C_{s \mathrm{e} r}$ - value of newly input funds;

$F_{K}$ - total cost of capital investments after reconstruction.

- $\quad$ Grade of initial reliability:

$$
P_{0} \geq\left[P_{0}\right]
$$

- $\quad$ Allowable risk of collapse of structures $R$ :

$$
R(t) \leq[R] \text {. }
$$


Moreover, the more input restrictions, the higher the actual cost of the construction becomes.

To minimize the alleged damage, it is best to consider the risk of failure. The quantitative risk assessment allows to solve tasks for determining the risk of an accident, and the expected expected material losses, determined by the area of destruction. Approximate risk levels and the corresponding volumes of damage are determined by the exponential dependence [3]:

1. Collapse of the $1 \mathrm{st}$ rank $\left(R=10^{-5}\right)$ - the area of destruction of $100 \mathrm{~m}^{2} \leq \mathrm{S} \leq 240 \mathrm{~m}^{2}$. Collapse to 6 floors - leads to a disproportionate collapse along the perimeter or adjacent spans in all directions;

2. Collapse of the 2 nd rank $\left(\mathrm{R}=5 \cdot 10^{-6}\right)$ - the area of destruction is $240 \mathrm{~m}^{2} \leq \mathrm{S} \leq 360 \mathrm{~m}^{2}$. Collapse to 9 floors;

3. Collapse of the $3 \mathrm{rd}$ rank $\left(\mathrm{R}=10^{-6}\right)$ - the area of destruction $\mathrm{S} \geq 360 \mathrm{~m}^{2}$. Collapse more than 9 floors.

This approach is more justifiable from an economic point of view and does not lead to an understatement of the bearing capacity [2].

To find the value of the function, the range of admissible values is first chosen and the variable parameters are set. The values of the function are obtained by the method of search optimization using the criterion of minimum cost.

Consider a searching technique of the most favorable decision of optimization problem. We choose a parameter that has a large effect on the values of the function. Increasing its value, we fall into the permissible region of the function. After this parameter is released to the most acceptable value, the search continues with a change in the percentage of reinforcement on which the cost parameters depend. By reducing the discrepancy by constraints, we reach the upper limit of the allowed values, which is determined to a greater extent by the possible consumption of resources. The optimal value will be at the minimum point of the function at the boundary of the admissible region. The position of this boundary is determined by groups of constraints, including taking into account the risk of collapse of the structure.

Consider an example of optimization of a non-beam slab with the following initial parameters. The size of the plate cell $1_{1} \times l_{2}=600 \times 400 \mathrm{~cm}$, column cross-section $b_{C}=40 \mathrm{~cm}$, class of concrete B30, armature A500, protective layers of concrete $a_{1}=2 \mathrm{~cm}, a_{2}=1,7 \mathrm{~cm}$, load q=20 kN/m $\mathrm{m}^{2}$. Cost of concrete $\mathrm{C}_{1}=4000 \mathrm{rub} . / \mathrm{m}^{3}$, armature $\mathrm{C}_{4}=1.775 .000 \mathrm{rub} . / \mathrm{m}^{3}$.

Denote the boundary conditions. To ensure the load-carrying capacity of the assembly of columns with a flat slab, check the bearing capacity of the slab in the normal section for the action of the negative bending moment:

$$
M \leq M_{\text {ult }} \text {. }
$$

Check for the effect of transverse forces:

$$
Q \leq Q_{u l t} \text {. }
$$

Check for forcing:

$$
F \leq F_{b, u l t}+F_{s w, u l t}
$$

For units, the load-carrying capacity test must be followed:

$$
\frac{P^{\text {разр }}}{A_{b}+A_{s} n} \leq\left[R_{b e}\right] \text {. }
$$

where $R_{b e}$-strength of concrete.

For each zone, the condition for limiting the width of the opening of normal and diagonal cracks:

$$
a_{T i} \leq\left[a_{T}\right]
$$


as well as the condition of limiting the deflection:

$$
W_{i} \leq[W] \text {. }
$$

When calculating the slab in the operational stage, the consumption of concrete varies within the limits $0,4-0,7 \mathrm{~m}^{3} / \mathrm{m}^{2}$, consumption of armature $35-70 \mathrm{~kg} / \mathrm{m}^{2}$, therefore, the constraints on the material will have the form:

$$
\begin{gathered}
V_{b} \leq 0,7 \mathrm{~m}^{3} / \mathrm{m}^{2}, \\
G_{s} \leq 70 \mathrm{~kg} / \mathrm{m}^{2} .
\end{gathered}
$$

It is recommended to use a concrete class not higher B50.

$$
R_{b} \leq 27,5 \mathrm{MPa}
$$

The coefficient of renewal is assumed equal to

$$
\left[k_{n}\right]=0,7 \ldots 0,9
$$

The restriction on the stability of the building against the progressive collapse, which is ensured if for any element the condition:

$$
F^{\prime} \leq[S]
$$

where $F^{\prime}$ and $S$ respectively, the force in the structural element, found from the performed static calculation, and its design load-carrying capacity.

Determination of an acceptable level of risk

$$
P(F)<P_{a d m},
$$

where $P_{a d m}$ - allowable risk.

In conditions of man-caused hazards (technical risk), individual risk is considered acceptable if its value does not exceed $10^{-6}$.

Consider the zone of maximum moments. In the results obtained, we fix the value of the plate height and in the future we will only vary the reinforcement coefficient.

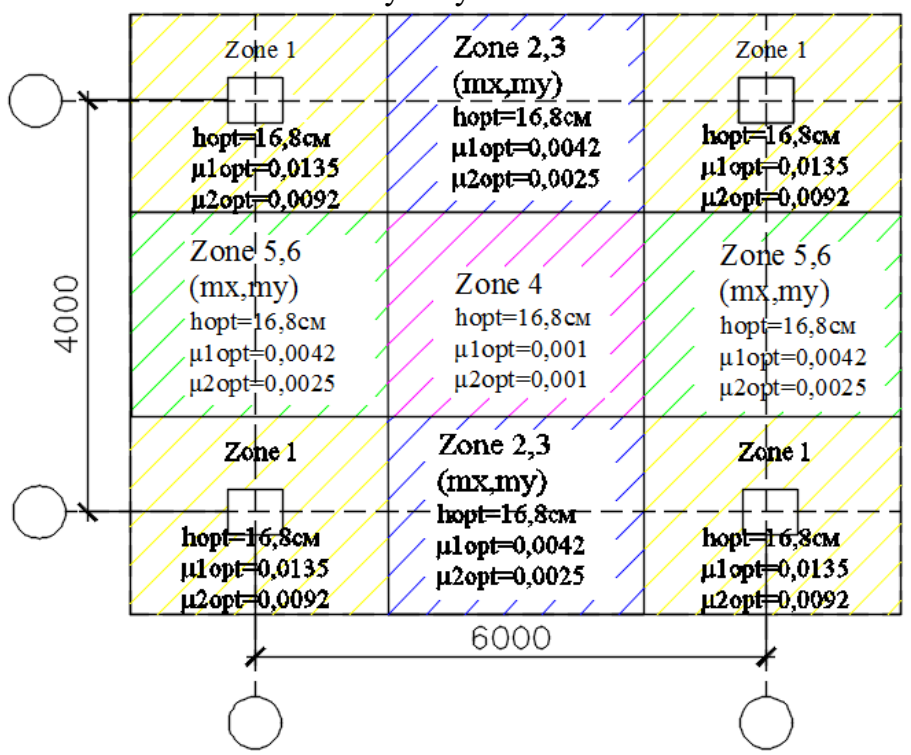

Fig. 1. Optimum scheme for reinforcing the zones of a non-beam slab

The calculation results with optimal parameters for different zones are schematically shown on the reinforcement plan (fig.1) 
We describe the method of searching for the optimal solution. Depending on the initial data and the adopted variable parameters, the stiffness characteristics for all zones are determined, the forces and their limit values are given for the given stiffnesses, the objective function $\mathrm{F}$ for any combination of plate parameters is calculated. Further, a small standard increment $\Delta x_{i}$ is introduced, and an additional factor that is the sensitivity factor of the value of the objective function at the optimum point to the resources:

$$
\Delta C=\frac{\Delta P}{\Delta F} .
$$

To reach the optimum point, $\Delta \mathrm{P}$ should be as large as possible and $\Delta \mathrm{F}$ smaller. The objective function $\mathrm{F}$ is computed and a generalized discrepancy $\mathrm{P}$ is found for the constraints. If the discrepancy is more than zero, it is eliminated and the final value of the cost function $\mathrm{F}$.

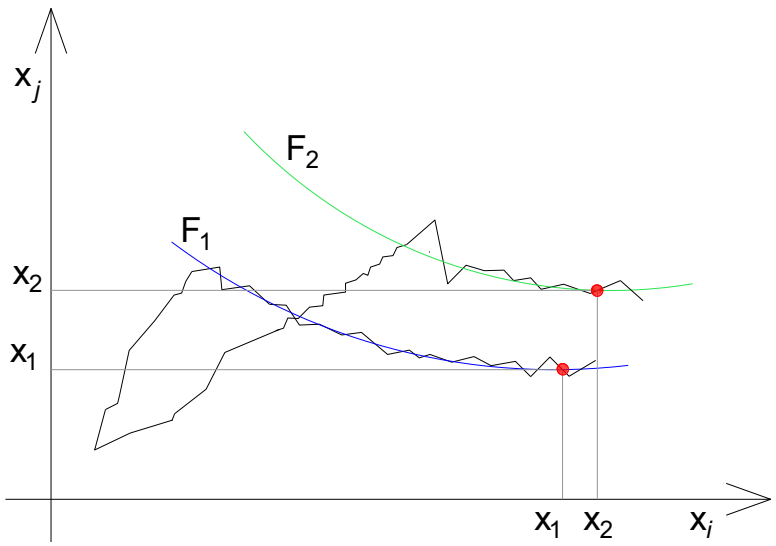

Fig.2. Scheme of searching for the optimum of the final element function. $\mathrm{x}_{1}$ and $\mathrm{x}_{2}$ - variable parameters of a reinforced concrete element.

In the calculations for progressive destruction, an instantaneous failure of one supporting element is assumed [4,5]. However, this is practically possible with explosive and shock effects accompanied by fires [6,7]. In case of fire in a loaded condition, the structures are exposed to high-temperature fire action, which changes the properties of concrete and reinforcement [8].

At the same time, dynamic loads begin to act on some structural elements, which, in combination with short-term fire effects, can be greater than the bearing capacity. This inevitably leads to destruction $[9,10]$. This kind of impact should be taken into account in a special combination of loads. In this case, it will be sufficient to satisfy only the safety requirements of the element or the structure as a whole [11].

As features of the calculation for progressive destruction, it is necessary to take into account the properties of materials and the design work under fire and dynamic influences [12]. The effect of a dynamic load application is characterized by a dynamic factor, that is, the ratio of concrete strength under dynamic loading to static strength [13-15]. Its value at normal temperatures is 1.8 , but at temperatures from $300^{\circ} \mathrm{C}$ and more it drops to $0,8-0,6$, which is 2,25-3 times smaller (fig. 3) [16].

With dynamic actions, the moments must be calculated:

$$
\begin{gathered}
M_{u, d}=\alpha_{m} R_{b d} b h_{0}^{2}, \text { when } \alpha_{m}=\xi(1-0,5 \xi) ; \\
\xi=\frac{R_{s d} A_{s}}{R_{b d} b h_{0}}, \\
R_{s d}=K_{d s} \cdot R_{s}
\end{gathered}
$$




$$
R_{b \mathrm{~d}}=K_{d b} \cdot R_{b}
$$

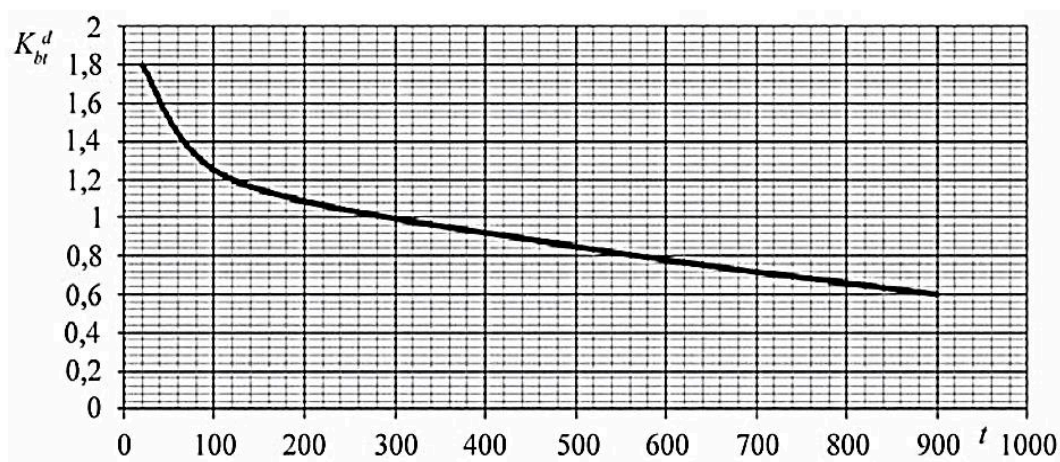

Fig.3 Graph of dependence of the coefficient of dynamism of concrete $K_{b t}^{d}$ on temperature

The dynamic coefficient depends on several indicators, including temperature, time and loading speed. And with an increase in the loading speed, its unevenness also increases, that is, certain areas of concrete prove to be highly overstressed. The dynamic load application changes the design design scheme. Based on the tests carried out, the analytical dependence of the dynamic strength of concrete on temperature at impact speed $0,4 \mathrm{~m} / \mathrm{s}$ :

$$
K_{d b}=1.27+9.97 \cdot 10^{-12} T^{4}-1.87 \cdot 10^{-8} T^{3}+1.10 \cdot 10^{-5} T^{2}-2.93 \cdot 10^{-3} T
$$

\section{Conclusion}

At the moment, in the calculations for progressive destruction in complexes and methodological instructions, the dynamic coefficient is taken to be greater than one [17-20], however, the experiments show a real decrease in its value when heated, which leads to a significant decrease in the bearing capacity of the element and an increase in the values of the first three terms of the cost function (1).

Comparing the functions $\mathrm{F}_{1}$ and $\mathrm{F}_{2}$ (fig. 2), we can say that the cost of manufacturing the element of the function $F_{2}$ will be higher than that found by the function $F_{1}$. However, the safety function $F_{2}$ will be the most profitable solution, because we reduce the risk of failure of the design. Verification calculations showed that $\mathrm{F}_{2}$ is more economical by 20 $30 \%$ compared to $\mathrm{F}_{1}$.

Using the cost function (1) when designing the cost function, taking into account the risk of loss, leads to an increase in the level of safety. As a rule, a decrease in the magnitude of the risk leads to an increase in the cost of constructing. On the other hand, increasing the risk may lead to the failure of structures in a shorter period. Therefore, the definition of the accepted value of risk is a very important task. The proposed objective function provides the most objective assessment of the costs for the projected facilities, taking into account the emergence of emergency situations. This approach will significantly improve the quality of design and avoid the occurrence of progressive destruction. 


\section{References}

1. DoD UFC: Design of buildings to resist progressive collapse, UFC 4-023-03, U.S. DoD. (2010).

2. A.G. Tamrazyan, E.A.Filimonova, Vestnic MSUCE 10 68-74 (2013).

3. A.G. Tamrazyan Izvestiya OSTU 4-16 107-108 (2007).

4. D.T. Pham, P. Buhan, C. Florence, J.-V. Heck, H.H. Nguyen ES 90 38-47 (2015).

5. H. Wanga, ${ }^{*}, Y . S u b$, Q.Zengc SEP 1 48-54 (2011).

6. A.G. Tamrazyan / A.G. Tamrazyan, L.A. Avetisyan TTP, Switzerland 638-640 (2014).

7. A.G. Tamrazyan ICSMIM 16-17, (2013).

8. MDV 21-2.2000.

9. A. Colombo and P. A Negro ES 27(8) 1164-1174 (2005).

10. C.T.Davie. H.LZhang, A.Gibson Comput Struct., 94-95 (3) 54-69 (2012)..

11. V.O.Almazov, A.I.Plotnikov, B.S.Rastorguev Vestnic MSUCE 2 15-20 (2011)

12. D.D. Capua, A.R. Fire Saf. 42(2) 139-49 (2007).

13. A.G. Tamrazyan, L.A. Avetisyan Vestnic MSUCE 10 14-23 (2013).

14. M. Bikhiet, F.Nasser, El-Shafey,Hany M. El-Hashimy Alexandria EJ 53 643-653 (2014)..

15. Y.S.Sin, J.E Park, J.Y. Kim Journal of the Korea Concrete Institute 2337 (2011).

16. A.G. Tamrazyan, L.A. Avetisyan ICC 4 24-28 (2014).

17. S.Kokot, A.Anthoine, P.Negro and G.Solomos ES 40 205-217 (2012).

18. Kwasniewski Leslaw 32 1223-1235 (2010).

19. Y.J. Kwon, N.H. Kim., J.Y. Lee, D.J. Kim, K.Harada, Proceedings of Fire Science and Engineering in Japan Association 282-283 (2010).

20. J.C. Mindeguia, H.Carré, P. Pimienta, La Borderie, In Rencontres Universitaires de Génie Civil. La Grande Motte. 1-2, (2006). 